\title{
Reconnaître la place des émotions dans le travail en groupe : étude exploratoire en éducation à l'entrepreneuriat
}

\author{
CAROLINE VERZAT \\ ESCP Business School, 79 avenue de la république, 75011 Paris \\ cverzat@escp.eu \\ NOREEN O'SHEA \\ ESCP Business School, 79 avenue de la république, 75011 Paris \\ noshea@escp.eu
}

\section{Résumé}

Les émotions sont-elles révélatrices de l'efficacité du travail en groupe ? Nous sommes parties d'une recherche initiale comparant deux dispositifs pédagogiques (autodirigé et dirigé) ayant pour objectif de développer la proactivité en entrepreneuriat (Verzat et al., 2016 ; Verzat et al., 2017). Nous en avons extrait des données mettant en relation la perception d'efficacité groupale et les émotions ressenties pendant les travaux de groupe. L'enquête a été menée en 2013, 2014 et 2015 à l'occasion d'un séminaire de 15 heures auprès de 281 étudiants en Master 1. Les résultats quantitatifs montrent des relations significatives entre la perception d'efficacité groupale (Guzzo et al., 1993) et deux groupes d'émotions positives et négatives. La recherche qualitative met en évidence les stratégies de régulation des apprentissages mobilisées par les étudiants dans le travail en groupe et leurs dimensions émotionnelles.

\section{Summary}

Do emotions reveal the degree of efficacy that groupwork can generate? Our research builds on a previous study that compared two pedagogical approaches - self-directed and teacher-directed learning- in developing the proactive attitude in entrepreneurial settings (Verzat et al., 2016; Verzat et al., 2017). For this study, we extracted the data which highlight the perception of group efficacy and emotions experienced during groupwork. The fieldwork was carried out in 2013, 2014 and 2015 with 281 master 1 level students involved in a 15hour seminar. The quantitative results of the present study show significant correlations between the perception of group efficacy (Guzzo et al., 1993) and two categories of positive and negative emotions. The qualitative results highlight the strategies adopted by the students to regulate group learning and its emotional dimensions.

\section{Mots-clés}

Émotions, travail en groupe, collaboration, stratégies de régulation 


\section{Key words}

Emotions, group work, collaboration, regulation strategies

\section{Introduction}

Reconnaitre ses propres émotions et celles d'autrui est l'un des piliers de la communication non violente permettant de résoudre des conflits (Rosenberg, 1999). Pour Ekman (2003), les messages émotionnels sont des moyens universels permettant de décrypter la capacité des humains à coopérer entre eux. Pourtant les pédagogies actives prônant la collaboration efficace au sein des groupes étudiants affichent une certaine ambivalence vis-à-vis des dimensions émotionnelles. Par exemple, le manuel sur l'apprentissage coopératif à destination des enseignants (Peyrat-Malaterre, 2011) montre d'un côté l'importance de l'affectivité au sein des groupes (p. 32) et souligne abondamment le rôle de la confiance, du respect et plus généralement des attitudes positives dans la mise en place et le maintien de la coopération au sein des groupes d'apprenants (p. 69-70). Mais d'un autre côté, l'ouvrage souligne les objections des enseignants se méfiant des groupes trop joyeux parce qu'ils n'apprendraient pas grand-chose (p. 20) et présente les dérives potentielles lorsqu'il y a trop d'affinité entre les membres des groupes risquant d'entraîner un relâchement dans le travail (p. 71) lorsque les modalités relationnelles l'emportent sur les processus socio-cognitifs (p. 82),

Le dilemme ne semble pas facile à résoudre car les recherches sur les émotions vécues au cours des processus d'apprentissage et leurs effets sont encore fragmentées et incomplètes (Pekrun et al., 2007). S'appuyant sur les avancées de Pekrun (2006), Cosnefroy (2011) propose un cadre théorique qui permet de prendre en compte les différentes émotions présentes à toutes les étapes de l'apprentissage autorégulé. Mais la relation entre les stratégies de régulation de l'apprentissage (cognitives, métacognitives et motivationnelles), et les dynamiques émotionnelles n'y est pas analysée systématiquement dans le contexte d'un travail en groupe. D'autre part, ce cadre théorique sous-tend une posture spécifique de la part de l'enseignant, à savoir la facilitation d'un apprentissage autodirigé (Knowles, 1975) ou autorégulé (Zimmerman, 1990). De son côté, Heutté (2017) propose un modèle heuristique analysant les conditions d'apprentissage liées aux émotions vécues dans les situations de travail en groupe d'apprenants. Mais son cadre de réflexion relié au concept de «flow» de Csikszentmihalyi $(1990,2014)$ se réfère à l'expérience optimale d'apprentissage, qui ne rend pas compte de toutes les émotions vécues. De plus, son modèle n'examine pas en détail les processus de régulation du travail en groupe et analyse de manière très globale les interactions entre les apprenants.

La question de départ de cette recherche est donc d'explorer dans quelles mesures différentes émotions sont associées à des processus d'apprentissage efficaces dans le contexte d'un travail en groupe collaboratif. Plus précisément, nous cherchons à investiguer trois questions : Tout d'abord quelles émotions sont associées à l'efficacité perçue par les apprenants selon les différents buts possibles du travail en groupe ? Deuxièmement quelles émotions sont associées aux stratégies de régulation de l'apprentissage (motivationnelles, cognitives ou métacognitives) mises en œuvre au sein du groupe ? Enfin dans quelle mesure, le choix d'un dispositif dirigé ou autodirigé influe-t-il sur les relations entre émotions et efficacité du travail en groupe ? 
Notre contribution est de nature exploratoire. Elle mesure la relation entre l'efficacité groupale perçue et un ensemble d'émotions positives et négatives ressenties par les étudiants au cours d'un travail de groupe. Cette mesure a été effectuée auprès de trois promotions d'étudiants en $1^{\text {re }}$ année de Master en management vivant un travail de groupe dans le cadre d'un séminaire de sensibilisation à l'esprit d'entreprendre de 15 heures. Deux dispositifs pédagogiques (dirigé / autodirigé) ont été testés. La recherche montre des corrélations significatives entre la perception d'efficacité groupale (Guzzo et al., 1993) et des émotions positives (joie, plaisir, fierté et excitation) et de façon inverse des émotions négatives (irritation, colère et épuisement). Elle montre que l'approche pédagogique influe aussi sur ces émotions et met en évidence différents types de stratégies mobilisées par les étudiants associées à ces émotions dans les deux types de dispositifs.

Notre article est structuré en quatre parties. La première présente notre cadre théorique et précise les objectifs de recherche qui en découlent. La deuxième explique la méthodologie choisie. La troisième partie présente les résultats. La quatrième partie discute ces résultats.

\section{Questionnement théorique sur les émotions dans la collaboration au sein des groupes d'apprentissage}

Trois concepts principaux sont articulés dans notre recherche : l'apprentissage autodirigé (ou autorégulé), les émotions associées aux processus de régulation des apprentissages et les processus de collaboration dans le travail en groupe. Après avoir présenté l'articulation progressive des trois concepts, nous proposerons la formulation de nos questions de recherche.

\subsection{L'apprentissage autodirigé / autorégulé}

Carl Rogers suggère dans son célèbre livre Freedom to learn (1969) que l'on peut stimuler à la fois l'autonomie et la joie d'apprendre et que cela est fortement lié à la posture de l'éducateur. Cette orientation s'incarne particulièrement dans les principes pédagogiques directeurs de l'apprentissage autodirigé (Knowles, 1975) ou autorégulé (Zimmerman, 1990, Cosnefroy, 2011). Dans cette approche, les étudiants choisissent par eux-mêmes leur propre objectif d'apprentissage ainsi que les stratégies et activités pour l'atteindre. Les enseignants ont la tâche de faciliter ces choix et d'aider les étudiants à effectuer des travaux avec un degré de guidage limité. À partir de sa revue de synthèse sur l'apprentissage autorégulé, Cosnefroy (2011) affirme que la participation active des apprenants au processus d'apprentissage a pour effet d'augmenter à la fois la performance dans le travail, le bien-être des apprenants et la réussite dans les études. De même Carré et al. (2010) soulignent l'intérêt de créer des environnements d'apprentissage autodirigé afin d'augmenter la motivation des apprenants au départ, de les aider à maintenir leur effort, ce qui les conduit à réussir et s'attribuer in fine le succès. Créer un tel environnement consiste pour l'enseignant à donner le plus de contrôle possible aux apprenants tout au long du processus d'apprentissage : les faire participer à la définition des buts, leur laisser contrôler la mise en œuvre des activités et favoriser l'auto-évaluation. Cette approche s'oppose à une approche dirigée où l'enseignant contrôle l'ensemble des étapes de la définition des objectifs d'apprentissage à l'évaluation. 
Cosnefroy (2011) propose un modèle unifié des mécanismes de régulation dont il est question du côté des apprenants. Il définit une taxonomie comportant quatre séries de stratégies mobilisées par les apprenants (cognitives, métacognitives, volitionnelles et défensives) afin de poursuivre plusieurs objectifs entrant parfois en conflit (apprendre mais aussi protéger l'estime de soi). Les stratégies cognitives (répéter, mémoriser, comprendre, transformer, concrétiser, créer des liens...) et métacognitives (s'orienter au départ, planifier les tâches d'apprentissage, gérer leur déroulement, évaluer le résultat puis élaborer les enseignements in fine) ont pour fonction de traiter les informations. Les stratégies volitionnelles ont pour fonction de protéger l'intention d'apprendre. Elles consistent dans le contrôle de l'attention (ou de désengagement provisoire), dans le contrôle de la motivation et des émotions et dans le contrôle du contexte d'apprentissage (espace de travail, ressources disponibles, organisation du temps). Enfin les stratégies défensives (au départ, éviter la confrontation à l'échec ou garantir le succès par exemple en se donnant des buts peu exigeants ou bien, après la tâche, éviter l'auto-évaluation ou les comparaisons menaçantes) ont pour fonction de protéger l'estime de soi. Cette taxonomie est précieuse parce qu'elle permet d'examiner la variété des émotions éprouvées par les étudiants lorsqu'ils mobilisent ces différentes stratégies.

\subsection{Les émotions dans l'apprentissage}

Dans les conceptions classiques de la cognition héritées de Descartes séparant l'esprit du corps, l'activité mentale rationnelle est séparée de l'émotion, perçue comme une pulsion biologique source de biais irrationnels. Mais de nombreux travaux récents à partir des travaux de Damasio (1995) sur le cerveau proposent une vision plus unifiée dans laquelle la perception des émotions interagit avec le raisonnement (Barth, 2013). Derouesné (2011) précise les deux courants (théorie psycho-évolutionniste / théorie de l'évaluation) qui discutent le statut des émotions par rapport à la cognition. Nous situons notre travail dans le courant de l'évaluation (appraisal) qui décrit l'émotion comme «le produit émergent du traitement d'un certain nombre de paramètres de l'événement-stimulus : nouveauté, soudaineté, caractère plaisant ou déplaisant, implication du Soi (agentivité, c'est-à-dire conscience que c'est le sujet lui-même qui est à l'origine de l'action), facilitation ou non de l'obtention des objectifs, congruence par rapport aux objectifs et aux attentes, potentiel de contrôle (coping), probabilité d'efficacité dans la situation, compatibilité avec les valeurs individuelles et sociales » (Scherer, 2009 cité par Derouesné, 2011, p. 73). Les émotions se distinguent des sentiments (feelings) et des humeurs $(\operatorname{moods})^{1}$.

De nombreuses recherches récentes montrent que les émotions sont importantes dans l'acte d'apprendre (Schutz et Pekrun, 2007 : Pekrun et al., 2007 ; Hinton et al., 2008 ; Valiente et al. 2012). Cosnefroy (2011) s'appuie sur la classification des émotions en contexte d'apprentissage de Pekrun (2006) qui prend en compte trois dimensions : leur valence (négative-déplaisante ou

\footnotetext{
${ }^{1}$ Contrairement aux humeurs qui désignent des états diffus sans relation avec un objet ou une situation spécifique, les émotions sont déclenchées par un stimulus-événement précis et ont une durée brève. Elles s'opposent également aux sentiments qui correspondent à une tendance à établir une relation prolongée positive ou négative vis-à-vis d'une personne ou d'un objet spécifique.
} 
positive-plaisante), le degré d'activation ${ }^{2}$ et le moment d'apparition (avant, pendant ou après le déroulement de la tâche). Certaines émotions sont liées à un moment précis (par exemple la fierté une fois la tâche accomplie) alors que d'autres sont susceptibles d'apparaître à différents moments (par exemple le plaisir peut être éprouvé pendant l'activité mais aussi par anticipation). Le Tableau 1 reproduit les combinaisons observables à partir de ces trois critères.

Tableau 1 : classification des émotions en contexte d'apprentissage selon Pekrun (2006) cité par Cosnefroy (2011, p. 67)

\begin{tabular}{|c|c|c|c|}
\hline & $\begin{array}{l}\text { Émotions prospectives } \\
\text { (avant l'activité) }\end{array}$ & $\begin{array}{ll}\text { Émotions pendant } & \text { le } \\
\text { déroulement } & \text { de } \\
\text { l'activité } & \end{array}$ & $\begin{array}{l}\text { Émotions rétrospectives } \\
\text { (après l'activité) }\end{array}$ \\
\hline Activation plaisante & $\begin{array}{l}\text { Espoir } \\
\text { Plaisir }\end{array}$ & Plaisir & $\begin{array}{l}\text { Fierté } \\
\text { Gratitude }\end{array}$ \\
\hline Activation déplaisante & Anxiété & $\begin{array}{l}\text { Colère } \\
\text { Anxiété } \\
\text { Frustration }\end{array}$ & $\begin{array}{l}\text { Honte } \\
\text { Culpabilité } \\
\text { Colère }\end{array}$ \\
\hline Désactivation plaisante & & & Soulagement \\
\hline $\begin{array}{l}\text { Désactivation } \\
\text { déplaisante }\end{array}$ & Désespoir & $\begin{array}{l}\text { Ennui } \\
\text { Désespoir }\end{array}$ & \\
\hline
\end{tabular}

Cosnefroy (2011, p. 66-67) explique que les émotions sont associées au niveau de contrôle exercé ou attendu sur l'activité et à la valeur perçue de cette activité. Dans la phase préparatoire de l'apprentissage, quand il s'agit d'orienter la tâche (activer des savoirs antérieurs, identifier les contraintes et les ressources, comprendre le problème à résoudre...) ou de planifier les tâches d'apprentissage (chercher des informations, relire ses notes, définir un but à atteindre, établir un plan détaillé, allouer des ressources en temps) des attentes de contrôle fortes et une valeur forte favorisent l'émergence d'un plaisir anticipateur. En revanche si l'apprenant a une perception de valeur forte mais une perception de contrôle faible, il risque de ressentir du désespoir. L'intensité des attentes fait aussi changer la nature des émotions. Dans cette phase préliminaire si les attentes sont modérées, c'est-à-dire que le résultat est incertain, c'est plus de l'espoir que du plaisir qui est éprouvé, et moins du désespoir que de l'anxiété. Cosnefroy ajoute que la nature du but perçu (de performance ou de maîtrise) influe sur la perception de valeur et de contrôlabilité qui sont à la racine des émotions éprouvées selon Pekrun (2006).

Pendant le déroulement de l'activité, l'association entre une forte valeur et une forte perception de contrôle génère du plaisir. Mais si l'activité suscite l'intérêt mais demande un effort trop élevé, elle génère de l'anxiété. Si elle demande un effort rebutant (contrôle élevé, valeur faible) elle génère de la colère. Si elle a peu de valeur et apparaît peu contrôlable, elle fait naître de la frustration. L'ennui apparaît dans les activités à valence neutre et contrôle modéré, ou bien

\footnotetext{
${ }^{2}$ Les émotions produisent une activation plus ou moins intense du système neuro-végétatif : accélération du pouls, de la respiration, sueurs, frissons, nausées...
} 
quand les capacités exigées sont élevées (attentes de contrôle fortes), mais la valeur perçue est faible.

Selon Pekrun (2014), les émotions positives et négatives associées à l'exécution d'une tâche ont un impact profond sur l'apprentissage et la performance des étudiants. Les émotions positives, par exemple la joie ressentie dans l'acte d'apprendre ont quatre effets : elles permettent de mobiliser l'attention, d'augmenter la motivation, de susciter la créativité et la flexibilité, ce qui facilite la régulation de l'apprentissage. D'un autre côté, les ressentis d'anxiété, de honte, d'ennui ou de colère réduisent l'attention portée à la tâche ainsi que la motivation intrinsèque des étudiants. Toutefois, un degré modéré d'émotions négatives peut également stimuler les étudiants pour éviter l'échec (désengagement et stratégies d'évitement). D'une manière générale, Cosnefroy (2011, p. 69) suggère d'étudier la manière dont les émotions se succèdent dans un épisode d'apprentissage car «il est sans doute réducteur de penser que les émotions négatives sont " mauvaises 》 et les émotions positives " bonnes 》 pour l'apprentissage. [...] C'est vraisemblablement un enchaînement dynamique d'émotions positives et négatives qui, dans certaines conditions et dans certains contextes, produit des effets éventuellement positifs sur l'apprentissage ».

\subsection{Les émotions dans le travail collaboratif en groupe d'étudiants}

Les émotions sont aussi liées aux buts sociaux présents dans la situation d'apprentissage comme par exemple, le besoin d'appartenance au groupe, l'affirmation de soi ou la production de résultats socialement désirables. La colère a été ainsi souvent associée au fait de subir une injustice par rapport aux autres étudiants (Boekaerts, 1994). À l'inverse. la présence de buts sociaux au-delà des buts de maîtrise ou de compétence peut renforcer la désirabilité d'une tâche, la faisant apparaitre plus stimulante et donc plus plaisante, même si elle n'est pas associée à un effort élevé sur le plan cognitif. Par ailleurs, Pekrun et al. (2002) mettent en évidence des émotions typiquement sociales différentes des émotions liées à l'accomplissement de la tâche académique. Les émotions typiquement sociales positives sont la gratitude, l'empathie, l'admiration, l'amour et la pitié. Les émotions négatives sont la colère, la jalousie et l'envie, le mépris, l'antipathie et la haine.

Les buts sociaux sont particulièrement développés dans les activités de groupe, notamment dans les situations d'apprentissage par problème et par projet qui exigent la collaboration effective des étudiants (Johnson et Johnson, 1991, 1998). Selon Roschelle et Teasley (1995) collaborer suppose de construire un espace-problème partagé, de définir ensemble des objectifs, de partager les connaissances disponibles et de s'accorder sur les tâches à mener pour résoudre le problème. On peut donc faire l'hypothèse que les processus de régulation associés aux stratégies volitionnelles et défensives y sont fortement mobilisés. La taxonomie de Cosnefroy (2011, p. $94,95,109)$ recense les stratégies suivantes. Du côté du soutien à l'apprentissage : renforcement des buts motivants (performance, maîtrise, intérêt intrinsèque de la tâche, utilité sociale perçue, récompenses), soutien du sentiment d'efficacité personnelle (par exemple activer des souvenirs de réussite, fractionner la tâche en sous-tâches, auto-encouragement), contrôle émotionnel (détaillé plus loin), contrôle de l'environnement. Du côté défensif : éviter la confrontation à l'échec lorsque les résultats sont compromis ou lorsque la tâche risque d'avoir des 
conséquences négatives dans les relations interpersonnelles (peine, conflit, perte de l'estime d'autrui), garantir le succès en se donnant des buts peu exigeants, en trichant ou en affichant un perfectionnisme défensif, procrastiner afin d'éviter un échec humiliant ou de simplement limiter sa charge de travail, et après la tâche, éviter l'auto-évaluation ou encore s'attribuer des évaluations auto-complaisantes, éviter les comparaisons menaçantes.

Les stratégies de contrôle émotionnel (ou régulation des émotions) consistent à induire ou maintenir des états ressentis positivement et à inhiber des états ressentis négativement. Elles semblent particulièrement importantes pour faciliter l'action et l'atteinte des buts communs. D'abord à cause de l'excitation physiologique et du partage social des émotions. Mais aussi et surtout parce qu'elles peuvent avoir plusieurs fonctions sociales : se conformer aux normes comportementales du groupe, éviter la confrontation et la désapprobation des autres, ou encore donner une présentation de soi avantageuse (compétente, assertive, optimiste) entretenant l'estime de soi. Par ailleurs, les stratégies de contrôle émotionnel peuvent être reliées aux buts de production externes plus ou moins contrôlables. Si les buts sont excessifs, des stratégies d'ajustement (coping) ou d'évitement visent à réduire le stress lorsque les exigences internes ou externes menacent ou dépassent les ressources de l'individu.

La synthèse de Cosnefroy (2011) ne portant pas spécifiquement sur les contextes de travail en groupe, nous avons repéré deux contributions qui étudient les interactions entre émotions et apprentissage dans un environnement de travail en groupe.

En premier lieu, Heutté (2017) qui vise à comprendre «ce qui pousse certaines personnes à vouloir comprendre, travailler ou jouer avec d'autres ». Il explique que les apprenants peuvent «se sentir littéralement portés par le contexte collectif, boosté par le flow et le sentiment de vivre une expérience optimale d'apprentissage » (Heutté, 2017, p. 91). Le concept de flow ${ }^{3}$ de Csikszentmihalyi $(1990,2014)$ fait référence à un état d'absorption cognitive, caractérisé par une attention totale qui absorbe (focalise) entièrement les ressources cognitives au point que la notion du temps disparaît. Cette attention est associée à un ressenti de bien-être et de «fluidité telle qu'à aucun moment l'apprentissage ou la compréhension ne seront interrompus par une quelconque inquiétude concernant ce qu'il faut faire pour y parvenir ou ce que les autres pourraient en penser ». (Heutté, 2017, p. 89). Les conditions groupales expliquant le maintien de l'absorption cognitive consistent dans la qualité des relations entre les membres du groupe, l'acceptation des autres ainsi que la qualité des relations et l'acceptation par les responsables du dispositif d'apprentissage. Heutté a mesuré que ces quatre variables génèrent une meilleure perception de compétence individuelle et surtout groupale, laquelle influe directement sur l'absorption cognitive et sur le flow. Heutté suggère que l'émotion associée au fait d'appartenir à «une communauté d'apprenants où chacun se sent accepté parce qu'il estime que ses compétences et son expertise sont reconnues » joue un rôle central pour engager et maintenir l'effort dans l'apprentissage : «l'émotion ressentie (cf. le flow) à ce moment-là, constitue l'un des moteurs de la persistance : si apprendre est rarement une partie de plaisir, comprendre (faire comprendre, être compris) peut être totalement jubilatoire » (Heutté, 2017, p. 92-93).

\footnotetext{
${ }^{3}$ La majorité des chercheurs conserve le terme anglais « flow ». Heutté propose la traduction française suivante « expérience optimale d'apprentissage » ou encore « autotélisme-flow ».
} 
La conjonction d'une qualité d'apprentissage et d'une qualité des relations se traduisant par une émotion positive partagée n'est malheureusement pas systématique. L'observation fine par auto-confrontation croisée réalisée par Foliard et Le Pontois (2017) auprès d'une équipe projet d'étudiants en entrepreneuriat montre que l'atteinte des buts d'apprentissage et de performance peut être associée à un ressenti très négatif. Ce projet avait été reconnu comme un succès à tous les niveaux en termes d'acquisition de compétences entrepreneuriales et de création de valeur ajoutée. Mais ce résultat avait été obtenu au prix d'une forte détérioration des relations entre les étudiants de l'équipe. L'explication de ce paradoxe est fournie grâce à l'examen des stratégies de coping menées par les étudiants pour gérer les tensions et conflits de rôles entre les étudiants. Deux typologies de stratégies de coping sont utilisées pour les analyser. Celle de Côté (2013) distingue les stratégies positives ou négatives selon la contrôlabilité de la situation, tandis que celle de Ionescu et al. (1997) distingue les mécanismes de défense évitant ou vigilant centrés sur les émotions ou sur le problème. Dans le cas observé, un étudiant a joué un rôle social mobilisant des stratégies de coping positives de nature émotionnelle (réévaluation positive) et vigilant (recherche de soutiens extérieurs). Un autre étudiant « impliqué à $600 \%$ » a pris le rôle d'organisateur et de visionnaire mobilisant des stratégies positives, vigilantes et centrées sur le problème en ce qui concerne le projet mais négatives en ce qui concerne le groupe (par exemple donner des buts irréalistes et déresponsabiliser les autres). Les trois autres étudiants ont adopté des stratégies défensives d'évitement. L'un d'eux s'est mis à l'écart au milieu du projet afin d'éviter la confrontation avec l'organisateur-visionnaire portant une vision du projet qu'il jugeait trop agressive. Les deux autres étudiants ont adopté une stratégie de lâcher-prise vis-àvis du sens général du projet, assurant seulement la partie des tâches qu'ils pouvaient contrôler et renonçant à des compétences qu'ils avaient pourtant envie de développer. La combinaison de l'ensemble de ces stratégies a permis au groupe de tenir les buts de production et d'apprentissage, mais elle n'a pas suffi pour tenir les enjeux sociaux de l'équipe.

\subsection{Synthèse et objectifs de recherche}

Notre question de départ partait de l'ambivalence perçue chez les enseignants à propos des émotions lors du travail en groupe. L'efficacité du groupe est-elle dépendante ou indépendante des émotions ressenties par les étudiants?

Notre revue de littérature nous a permis de mettre en évidence trois éléments de réponse.

1. Les émotions semblent jouer un rôle fort dans la perception d'efficacité collective des groupes d'apprentissage. Selon le modèle théorique de Heutté (2017), le sentiment d'efficacité collectif renforce le contrôle cognitif et l'immersion dans l'apprentissage entraînant un sentiment de bien-être à condition que le collectif soutienne les besoins psychologiques de base du groupe (qualité interpersonnelle des relations et sentiment d'acceptation entre pairs et avec les responsables du dispositif). L'observation de Foliard et Le Pontois (2017) montre à l'inverse que lorsque les étudiants ne parviennent pas à cette qualité de relation, ils ont des ressentis contrastés. Toutefois cela ne les empêche pas de tenir certains buts du projet. La diversité émotionnelle pourrait donc être liée à la multiplicité des buts poursuivis par les différents étudiants du groupe 
(apprentissage, production ou buts sociaux) et des rôles qu'ils prennent plus ou moins consciemment, mobilisant des stratégies d'ajustement différenciées.

2. Par ailleurs, selon les modèles de Cosnefroy (2011) et de Pekrun (2002, 2006, 2014), les stratégies d'autorégulation de l'apprentissage (cognitives, métacognitives, volitionnelles et défensives) génèrent une variété d'émotions au cours du temps en fonction de la gestion du couple contrôle-valeur lié aux buts poursuivis par les étudiants.

3. Enfin les travaux de Carré et al. (2010) suggèrent que les buts et les modalités de contrôle de l'apprentissage varient en fonction du dispositif pédagogique choisi par l'enseignant (autodirigé / dirigé).

Il reste néanmoins des zones d'ombre considérables. En effet le modèle de Heutté se focalise sur la seule émotion du flow et n'observe pas les stratégies de régulation mobilisées par les étudiants. L'observation de Foliard et Le Pontois porte majoritairement sur les stratégies défensives et n'est pas précise sur les émotions ressenties. Enfin la typologie de Cosnefroy (2011) la plus complète en termes d'intégration entre stratégies d'apprentissage et émotions est pensée au niveau individuel. Nous ignorons donc le rôle des émotions positives et négatives générées par les stratégies d'apprentissage adoptées par les étudiants en situation de travail en groupe collaboratif. Notre recherche vise donc à :

1. identifier les émotions positives et négatives ayant un effet sur la perception d'efficacité collective.

2. explorer les stratégies d'apprentissage associées aux différentes émotions au-delà de l'absorption cognitive associée au flow.

3. vérifier quelles émotions sont associées aux buts poursuivis par les étudiants et au type de dispositif pédagogique choisi par l'enseignant (autodirigé / dirigé).

Ces objectifs sont particulièrement pertinents dans le domaine de la formation à l'entrepreneuriat. Certains auteurs ont montré qu'une intensité émotionnelle couplée à une dynamique de groupe efficace renforce l'apprentissage dans les projets (Arpiainen et al., 2013 ; Pittaway et Cope, 2007). Mais ils ne rentrent pas dans le détail des émotions impliquées, ni dans l'examen des processus d'apprentissage mobilisés au cours des projets. C'est l'objet de notre recherche qui porte sur un séminaire de formation à l'entrepreneuriat où deux dispositifs ont été testés sur la même population, faisant ainsi varier les buts des apprenants et le type de pédagogie de l'enseignant.

\section{Méthodologie}

\subsection{Les sujets et les approches étudiées}

Le contexte est un séminaire de 15 heures pour des étudiants de $1^{\text {re }}$ année de master dans une Business School française. L'objectif pédagogique est de développer l'esprit d'entreprendre. Deux dispositifs pédagogiques différents ont été proposés par les trois mêmes enseignants à trois promotions : une approche d'apprentissage autodirigé en 2013 (103 étudiants, dont 53 hommes et 50 femmes) et 2014 (115 étudiants, dont 61 hommes et 54 femmes) et une approche dirigée par l'enseignant à un groupe de contrôle composé de 63 étudiants en 2015 (dont 34 
hommes et 29 femmes). Le Tableau 2 résume les principes pédagogiques suivis et leur mise en œuvre dans les deux approches.

Tableau 2 : Les approches pédagogiques testées

\begin{tabular}{|c|c|c|}
\hline & $\begin{array}{c}\text { Dispositif Autodirigé } \\
\text { (promotions } 2013 \text { et 2014) }\end{array}$ & $\begin{array}{c}\text { Dispositif dirigé par l'enseignant } \\
\text { (promotion 2015) } \\
\end{array}$ \\
\hline \multirow{3}{*}{$\begin{array}{l}\text { Principes } \\
\text { pédagogiques }\end{array}$} & Choix maximal par les étudiants & Peu de choix par les étudiants \\
\hline & Enseignant facilitateur & Enseignant guide expert et directif \\
\hline & $\begin{array}{l}\text { Focus sur l'expérience et le sens donné } \\
\text { par le groupe d'étudiants }\end{array}$ & $\begin{array}{l}\text { Focus sur les connaissances académiques et } \\
\text { les compétences de recherche }\end{array}$ \\
\hline $\begin{array}{l}\text { Objectif } \\
\text { d'apprentissage }\end{array}$ & $\begin{array}{l}\text { Expérimenter l'esprit d'entreprendre par } \\
\text { le biais d'expériences stimulantes } \\
\text { choisies librement par le groupe } \\
\text { d'étudiants }\end{array}$ & $\begin{array}{l}\text { Définir l'esprit d'entreprendre grâce à la mise } \\
\text { en œuvre de mini-recherches scientifiques à } \\
\text { partir de concepts de la littérature }\end{array}$ \\
\hline $\begin{array}{l}\text { Formation des } \\
\text { groupes } \\
\text { d'étudiants }\end{array}$ & $\begin{array}{l}\text { Groupes d'affinité choisis librement par } \\
\text { les étudiants }\end{array}$ & Groupes aléatoires imposés par l'enseignant \\
\hline $\begin{array}{l}\text { Activités } \\
\text { d'apprentissage en } \\
\text { groupe }\end{array}$ & $\begin{array}{l}\text { Négociation de contrat d'apprentissage } \\
\text { entre les groupes et l'enseignant avec } \\
\text { suivi ad hoc selon les choix et les besoins } \\
\text { des étudiants : } \\
\text { a. choix et réalisation d'un projet libre } \\
\text { signifiant l'esprit d'entreprendre } \\
\text { pour les étudiants (créativité } \\
\text { encouragée) ; } \\
\text { b. choix de l'heure de rencontre ; } \\
\text { c. activités autonomes liés au projet } \\
\text { choisi. }\end{array}$ & $\begin{array}{l}\text { Activités conçues, guidées et contrôlées pas à } \\
\text { pas par les enseignants dans cet ordre : } \\
\text { 1. lectures académiques sur l'esprit } \\
\text { d'entreprendre (lectures imposées); } \\
\text { 2. élaboration d'un mini projet de recherche } \\
\text { (choix guidé); } \\
\text { 3. faire des entrevues ou des questionnaires ; } \\
\text { 4. analyse guidée des résultats. }\end{array}$ \\
\hline \multirow{2}{*}{$\begin{array}{l}\text { Rythme des } \\
\text { activités }\end{array}$} & Réunions en classe (45mn) & \multirow{2}{*}{$\begin{array}{l}\text { Toutes les sessions ( } 3 \mathrm{~h} \text { ) dans la salle de classe } \\
\text { et toujours en présence de l'enseignant }\end{array}$} \\
\hline & $\begin{array}{l}\text { Autres activités autonomes à l'extérieur } \\
(2 \mathrm{~h} 15)\end{array}$ & \\
\hline \multirow[t]{2}{*}{$\begin{array}{l}\text { Livrable à la fin du } \\
\text { séminaire }\end{array}$} & $\begin{array}{l}\text { Présentation créative par le groupe pour } \\
\text { impressionner toute la classe (grande } \\
\text { diversité: théâtre, vidéos, débats, jeux...) }\end{array}$ & $\begin{array}{l}\text { Présentation académique pour répondre aux } \\
\text { consignes précises des enseignants }\end{array}$ \\
\hline & $\begin{array}{l}\text { diversite: theatre, videos, débats, jeux...) } \\
\text { Présentation entièrement gérée par le } \\
\text { groupe }\end{array}$ & $\begin{array}{l}\text { Processus de présentation contrôlé par } \\
\text { l'enseignant }\end{array}$ \\
\hline Évaluation & $\begin{array}{l}\text { Auto-évaluations fondées sur des } \\
\text { critères négociés entre le groupe et } \\
\text { l'enseignant }\end{array}$ & $\begin{array}{l}\text { Notes attribuées par l'enseignant sur des } \\
\text { critères prédéfinis annoncés aux étudiants à } \\
\text { l'avance }\end{array}$ \\
\hline $\begin{array}{l}\text { Exercice réflexif } \\
\text { individuel }\end{array}$ & $\begin{array}{l}\text { Auto-réflexion guidée (questionnaire } \\
\text { semi-directif ouvert) sur l'expérience } \\
\text { d'apprentissage (processus et les } \\
\text { résultats) }\end{array}$ & $\begin{array}{l}\text { Auto-réflexion guidée (questionnaire semi- } \\
\text { directif ouvert) sur les résultats de la } \\
\text { recherche et de l'application à soi-même }\end{array}$ \\
\hline
\end{tabular}

Après le séminaire, les étudiants des trois promotions ont rempli un questionnaire de recherche identique en ligne afin d'évaluer notamment les émotions ressenties ainsi que la perception de l'efficacité des groupes de travail. Les trois promotions étaient réparties en classes de 20 à 30 étudiants encadrés par les mêmes trois enseignants, ce qui permet d'écarter l'hypothèse explicative d'un effet maître. 
Nous détaillons ci-dessous les mesures qualitatives et quantitatives réalisées ainsi que les techniques d'analyse utilisées.

\subsection{Mesures de la perception de l'efficacité groupale et des émotions}

Il existe plusieurs indicateurs de mesure de l'efficacité groupale. Bandura décrit l'efficacité collective comme la croyance partagée par les membres d'un groupe dans leurs capacités conjointes à organiser et à exécuter les plans d'action requis pour produire les objectifs communs à un niveau donné de réussite (Bandura, 1997, p. 447). Nous avons préféré utiliser la notion de puissance de groupe (group potency) qui rend compte d'une évaluation partagée et globale de la performance d'un groupe telle qu'elle est perçue par ses membres (Guzzo et al. (1993). Chaque item du questionnaire de Guzzo et al. (1993) est mesuré en utilisant une échelle de Likert à 6 points allant de 1 «pas du tout d'accord » à 6 « tout à fait d'accord ». La perception de chaque membre d'un groupe est la moyenne de ses scores aux 8 items ${ }^{4}$.

Nous avons mesuré les émotions à partir d'une liste composée de 14 émotions ${ }^{5}$ répertoriées par l'Emotion Research Group de Genève (2002), fer de lance du courant de l'évaluation (appraisal) dans l'étude des émotions, auxquelles nous avons ajouté 3 émotions décrites spécifiquement dans le domaine de l'entrepreneuriat, par Morris et al. (2010) à savoir l'excitation, l'angoisse et l'épuisement. Les étudiants ont évalué celles qu'ils avaient ressenties pendant le cours. Chaque émotion a été notée sur une échelle de 6 points avec un format de réponse de type Likert indiquant l'intensité de l'émotion ( 1 = pas du tout ressenti, 6 = très fortement ressenti).

Trois questions ouvertes ont été posées dans le questionnaire post séminaire afin de relier les perceptions émotionnelles aux buts perçus de l'exercice et aux stratégies d'apprentissage principales mobilisées tels qu'ils sont verbalisés par les étudiants en fin de parcours. 1) Qu'avez-vous appris personnellement au cours du séminaire Esprit d'entreprendre ? 2) Quels facteurs contribuent selon vous à expliquer ce résultat ? 3) Quels sentiments principaux ${ }^{6}$ avezvous éprouvés tout au long du séminaire (décrivez-les avec vos propres mots)?

\subsection{Méthodes d'analyse quantitative et qualitative}

La méthode d'analyse quantitative est basée sur des tests de corrélations entre les scores de perception individuelle de la perception d'efficacité groupale et les émotions déclarées ressenties par chaque individu. Afin de mesurer l'effet de la pédagogie sur cette relation, pour

\footnotetext{
${ }^{4}$ La traduction des 8 items de Guzzo et al. (1993) est la suivante : 1) Notre équipe a confiance en elle-même, 2) Notre équipe croit qu'elle peut devenir exceptionnellement efficace dans l'exécution d'un travail de haute qualité, 3) Notre équipe s'attend à être reconnue comme une équipe très performante, 4) Notre équipe sent qu'elle peut résoudre n'importe quel problème qu'elle rencontre, 5) Notre équipe crô̂t qu'elle est très productive, 6) Notre équipe peut accomplir une grande quantité de travail lorsqu'elle travaille fort, 7) Aucune tâche n'est trop difficile pour notre équipe, 8) Notre équipe s'attend à avoir beaucoup d'influence sur les autres équipes

${ }^{5}$ Les 14 émotions répertoriées par le questionnaire genevois d'appraisal sont la tristesse, la joie, la colère, l'anxiété, la surprise, la peur, l'irritation, la honte, le mépris, la culpabilité, le dégoût, le plaisir, le désespoir et la fierté. Cette liste est très proche des émotions décrites par Pekrun (2006).

${ }^{6} \mathrm{La}$ formulation «sentiments » est théoriquement inadéquate pour désigner des émotions (voir note $\mathrm{n}^{\circ} 2$ ). Mais dans le langage courant, les deux notions sont confondues. Le mot «sentiment» a été préféré ici afin d'éviter d'influencer les étudiants qui venaient de cocher des émotions dans une liste pré-suggérée.
} 
chaque échantillon (pédagogie autodirigée 2013+2014 / pédagogie dirigée 2015), nous avons effectué une classification des scores de perception d'efficacité groupale en 3 classes (faible, moyenne, forte $)^{7}$ et calculé les moyennes de ressenti sur chaque émotion dans chacune de ces 3 classes. Un diagramme en radar représente les déclarations de ressentis moyens par classe d'efficacité groupale pour toutes les émotions qui présentent une corrélation statistiquement significative.

La méthode des corrélations donne des indications dont il est difficile de tirer des relations de cause à effet. C'est pourquoi nous avons complété ce premier niveau d'analyse par l'exploration qualitative des verbatim afin d'interpréter les relations entre efficacité perçue, émotions ressenties, buts perçus du séminaire et stratégies de régulation des apprentissages mobilisées. L'analyse a été effectuée en plusieurs phases par les deux auteurs afin d'organiser le système de codage et d'assurer un degré d'accord satisfaisant. Nous avons d'abord divisé les verbatim en deux groupes selon le dispositif (autodirigé / dirigé). Puis pour les deux premières questions sur les enseignements du séminaire et sur les facteurs d'atteinte des résultats, nous avons utilisé la méthode de codage ouvert de Locke (2001) afin d'identifier les groupes de signification ressortant des expressions naturelles des étudiants. Ensuite les deux chercheurs ont confronté leurs catégories et corrigé leurs encodages afin d'obtenir un accord de $100 \%$ sur les catégories et sur les encodages. Puis ils ont comparé ces catégories à la littérature afin de produire des interprétations et se sont mis d'accord à nouveau sur les regroupements pertinents. En ce qui concerne les émotions déclarées librement, tous les verbatim exprimant explicitement une émotion ont été gardés tels quels. En revanche, lorsque les émotions n'étaient pas explicitement mentionnées, les deux chercheurs se sont mis d'accord sur une interprétation commune. Finalement, les poids relatifs de chaque catégorie d'interprétation ont été calculés à partir du nombre de verbatim de cette catégorie par rapport au nombre total des réponses à la question.

\section{Résultats}

Nous obtenons trois résultats en réponse aux questions de recherche posées.

\subsection{Résultat 1 - Les émotions sont reliées à la perception d'efficacité groupale}

Sur le plan quantitatif, le tableau 3 montre qu'il existe des corrélations significatives entre la perception de l'efficacité groupale perçue et les émotions déclarées.

\footnotetext{
${ }^{7}$ faible si $x \leq m-\sigma$, moyenne si $m-\sigma \leq x<m+\sigma$, forte si $x \geq m+\sigma$.
} 
Tableau 3 : corrélations entre perceptions d'efficacité groupale, sexe, pédagogie et émotions déclarées

\begin{tabular}{|c|c|c|c|c|c|c|c|c|c|c|c|c|c|c|}
\hline & & moyenne & écartype & 1 & 2 & 3 & 4 & 5 & 6 & 7 & 8 & 9 & 10 & 11 \\
\hline 1 & sexe & 1.5 & 0.5 & 1 & & & & & & & & & & \\
\hline \multirow[b]{2}{*}{2} & pédagogie : & & & & & & & & & & & & & \\
\hline & $\begin{array}{l}\text { autodirigée = } 1 \\
\text { dirigée=2 }\end{array}$ & 1.2 & 0.4 & -0.010 & 1 & & & & & & & & & \\
\hline \multirow{2}{*}{3} & efficacité & & & & & & & & & & & & & \\
\hline & groupale & 32.79 & 4.91 & 0.045 & 0.025 & 1 & & & & & & & & \\
\hline 4 & joie & 4.67 & 1.16 & 0.112 & $-0.274 * * *$ & $0.389 * * *$ & 1 & & & & & & & \\
\hline 5 & plaisir & 4.72 & 1.20 & 0.087 & $-0.352^{* * *}$ & $0.303^{* * *}$ & $0.721^{* * *}$ & 1 & & & & & & \\
\hline 6 & fierté & 4.38 & 1.30 & 0.065 & $-0.342^{* * *}$ & $0.337^{* * *}$ & $0.508^{* * *}$ & $0.567^{* * *}$ & 1 & & & & & \\
\hline 7 & excitation & 4.36 & 1.37 & 0.149 & $-0.334^{* * *}$ & $0.333^{* * *}$ & $0.628^{* * *}$ & $0.709 * * *$ & $0.607^{* * *}$ & 1 & & & & \\
\hline 8 & surprise & 4.09 & 1.44 & -0.036 & $-0.311 * * *$ & 0.150 & $0.338^{* * *}$ & $0.394^{* * *}$ & $0.223^{* * *}$ & $0.359^{* * *}$ & 1 & & & \\
\hline 9 & irritation & 1.99 & 1.34 & 0.088 & $0.183^{* *}$ & $-0.223 * *$ & $-0.167^{*}$ & $-0.164^{*}$ & -0.074 & -0.084 & -0.031 & 1 & & \\
\hline 10 & colère & 1.93 & 1.24 & -0.015 & $0.148^{*}$ & $-0.295 * *$ & $-0.195 * *$ & $-0.169^{*}$ & -0.025 & -0.132 & -0.001 & $0.600^{* * *}$ & 1 & \\
\hline 11 & épuisement & 2.21 & 1.36 & -0.030 & $0.200^{* *}$ & -0.063 & -0.058 & -0.058 & 0.034 & 0.013 & -0.077 & $0.354 * * *$ & $0.396 * * *$ & 1 \\
\hline$n=$ & $* * * p>0,001$ & $* p>0,01$ & ${ }^{*} p>0,05$ & & & & & & & & & & & \\
\hline
\end{tabular}

Il apparaît que le sexe n'a aucune influence ni sur l'efficacité groupale perçue, ni sur les émotions ressenties. La pédagogie ne fait pas non plus varier la perception d'efficacité groupale $(r=0,025$, non significatif). Les groupes ne semblent donc pas significativement plus efficaces dans une pédagogie ou dans l'autre, au regard des critères de Guzzo et al. (1993) qui portent essentiellement sur le résultat atteint. On pourrait penser que ceci est lié au fait que les deux situations pédagogiques ne sont pas si éloignées l'une de l'autre s'agissant d'un projet à réaliser en groupe. Mais nous verrons plus loin que l'examen qualitatif des émotions reliées aux buts et aux stratégies de régulation des apprentissages mobilisées amène à nuancer cette interprétation.

Quoi qu'il en soit, il apparaît que les émotions déclarées varient sensiblement avec l'efficacité groupale. Même si les corrélations sont modérées, elles sont nettement significatives. Les quatre émotions positives de la liste d'émotions utilisée (joie, plaisir, fierté, excitation) varient globalement ensemble et sont significativement plus importantes lorsque les étudiants perçoivent leur groupe comme efficace. À l'inverse, bien que dans une moindre mesure en termes statistiques, la colère et l'irritation ont tendance à être associées aux groupes les moins efficaces. Ces résultats sont confirmés mais nuancés par l'analyse qualitative des réponses à la question 3. Nous avons constaté une grande variété d'émotions reportées dans le tableau 4. 
Tableau 4 : codage des émotions exprimées en réponse à la question «Quels sentiments principaux avez-vous éprouvés tout au long du séminaire (décrivez-les avec vos propres mots) ? $\gg$.

\begin{tabular}{|c|c|c|c|c|c|}
\hline positives & & & négatives & & \\
\hline joie & 81 & $19 \%$ & culpabilité & 1 & $0 \%$ \\
\hline excitation & 52 & $12 \%$ & regret & 1 & $0 \%$ \\
\hline fierté & 40 & $9 \%$ & irritation & 12 & $3 \%$ \\
\hline espoir & 9 & $2 \%$ & colère & 10 & $2 \%$ \\
\hline plaisir & 64 & $15 \%$ & épuisement & 6 & $1 \%$ \\
\hline gratitude & 1 & $0 \%$ & frustration & 11 & $3 \%$ \\
\hline amour & 2 & $0 \%$ & déception & 11 & $3 \%$ \\
\hline passion & 3 & $1 \%$ & stress & 25 & $6 \%$ \\
\hline admiration & 1 & $0 \%$ & ennui & 10 & $2 \%$ \\
\hline soulagement & 11 & $3 \%$ & anxiété & 31 & $7 \%$ \\
\hline intérêt & 10 & $2 \%$ & desespoir & 3 & $1 \%$ \\
\hline total positives & 274 & $63 \%$ & peur & 13 & $3 \%$ \\
\hline neutres & & & pitié & 1 & $0 \%$ \\
\hline surprise & 10 & $2 \%$ & total négatives & 135 & $31 \%$ \\
\hline doute-confusion & 14 & $3 \%$ & & & \\
\hline total neutres & 24 & $6 \%$ & & & \\
\hline
\end{tabular}

total des émotions exprimées par 241 groupes $\quad 433 \quad 100 \%$

La joie, l'excitation, le plaisir et la fierté dominent mais il y a aussi des expressions significatives de soulagement et d'intérêt. Il n'est pas étonnant que les émotions positives soient dominantes, étant donné le biais de mémorisation des événements positifs démontré par Matlin et Stang (1978). Mais nous constatons aussi des expressions plus neutres de surprise ou de doute ainsi qu'une palette large d'émotions négatives : anxiété, peur, stress, irritation, frustration, déception, ennui et épuisement.

La joie et le plaisir sont surtout liés à la qualité du partage en équipe, ce qui entretient une forte motivation. «Pendant cette expérience, je me suis bien amusé. J'étais heureux de travailler avec une équipe motivée, unie et efficace. J'étais aussi content de voir l'enthousiasme que cette présentation a généré auprès des autres étudiants. » La joie peut aussi être liée à un sentiment de liberté du fait de la démarche pédagogique inhabituelle. "Nous avions l'opportunité de vraiment appliquer nos idées, de discuter, de transmettre notre vision de l'entrepreneuriat à travers un projet open source. Je l'ai adoré et je me suis senti très impliqué. " «Quelle joie d'avoir à faire quelque chose de concret! C'est tellement différent des études classiques. » L'excitation est liée au fait de travailler de manière créative en utilisant son imagination et aussi d'impressionner la classe. Mais cela n'est pas sans engendrer un certain stress. "J'ai vécu de l'excitation pendant le projet. Je me suis bien amusé en le présentant. Mais j'ai été stressé avant la présentation. Après, j'étais fier quand j'ai vu l'excellence de nos résultats. » La joie, le plaisir (au sens de satisfaction) et la fierté sont les émotions résultant des réussites perçues. «J'étais très fier du projet que nous avons fait parce que c'était un format libre. C'est nousmêmes qui avons choisi les interviewés. Après on a fait le montage. C'était fatiguant parce que 
c'est long de monter. Mais quelle récompense à la fin!». «Au début c'était angoissant. J'avais peur de ne pas réussir. À la fin, j'ai éprouvé un sentiment de satisfaction personnelle, de la fierté parce que nous avons réussi à engager les étudiants, à leur transmettre notre vision de l'entrepreneuriat. Nous avons reçu des feedbacks positifs des étudiants et du professeur. »

On voit qu'il y a rarement une seule émotion exprimée dans chaque verbatim (en moyenne 1,8 par personne). Certains ne font état que des émotions positives, d'autres uniquement négatives mais beaucoup font part d'émotions mélangées, rendant compte d'un vécu contrasté selon les différents moments du travail en équipe. Dans les verbatim à valence mixte, plusieurs formes de stress ou d'anxiété ont été identifiées. Au démarrage et pendant l'activité, le stress est lié au manque d'idées ou de compétences perçues : «J'étais anxieux à l'idée de ne pas trouver un projet. En fait, au début, on voulait faire une vidéo mais on n'avait pas les compétences. Finalement on a choisi un jeu, et même si c'était stressant de trouver les bonnes questions et de créer quelque chose de drôle pour nos pairs, nous avons enfin réussi quelque chose de valable. » Le stress et l'anxiété sont aussi très présents vers la fin de l'exercice au moment de présenter les résultats devant les pairs. "J'étais fier de ma présentation mais un peu anxieux de la réaction des autres. Et ensuite très heureux de voir qu'ils l'avaient appréciée. " Le soulagement apparaît lorsque la personne peut échapper à ce stress final : "Je dois admettre que j'ai été soulagé quand j'ai vu la personne qui a été choisie pour faire la présentation. C'était la meilleure pour représenter notre groupe parce qu'elle avait de très grandes compétences à l'oral. » Le soulagement peut aussi être lié au fait d'avoir surmonté l'épreuve : "J'étais stressé au début de la présentation parce que malgré le fait que je me sois beaucoup préparé, je ne pouvais pas prédire si la classe allait jouer le jeu. Comme ça a bien marché, j'ai ressenti du soulagement. »

Dans le groupe des émotions mixtes, certains récits mélangent fierté et frustration par manque de compétences. "J'étais très confiant et fier de notre équipe pendant toute la durée du projet. J'étais excité à l'idée de faire des entretiens et d'apprendre à coder les résultats. Quelquefois notre groupe était frustré parce que nous ne maîtrisions pas tous les paramètres de notre projet. » Mais le plus souvent les colères, irritations, frustrations et déceptions résultent des conflits au sein du groupe, en particulier de la difficulté à impliquer tous les membres : «J'étais très excité au début. J'ai bien aimé les discussions avec le prof. Mais en dehors du cours, j'ai ressenti de la frustration vis-à-vis des personnes qui ne contribuaient pas régulièrement au projet. » "J'ai ressenti de la colère vers la fin du projet quand il y avait des tensions au sein du groupe, j'ai lutté pour comprendre le sens de ces conflits. » "J'ai ressenti d'abord de l'excitation avant de réaliser que certains étudiants n'étaient pas du tout concernés par le projet. C'est là que j'ai ressenti le plus de colère et de stress. »

Certains groupes sont perdus au point de n'exprimer que des émotions négatives persistantes du début à la fin. Cela est lié à la fois au manque de sens de l'exercice et au manque d'aide au sein du groupe. "J'étais perdu au début parce que je ne comprenais pas ce qui était attendu. J'étais aussi anxieux parce que personne dans le groupe ne pouvait m'aider à comprendre. » «J'avais l'impression d'être initiée à faire de la recherche, or ça ne m'intéresse pas, j'aurais préféré rester dans le concret. » Le manque de compréhension au départ peut amener une sorte de désengagement tendant vers la neutralité émotionnelle. «En fait je ne dirais pas que j'ai 
ressenti beaucoup d'émotions pendant ce cours. Bien sûr parfois de la frustration, quand nous ne trouvions pas le temps de nous rencontrer avec le groupe en dehors des horaires du cours. Mais vis-à-vis du sujet ou pendant le cours, je n'avais pas de sentiment particulier. Je dirais que j'étais plutôt neutre, à part un sentiment de frustration et d'anxiété quand je ne comprenais pas l'objectif de ce travail. »

Le tableau 5 identifie les groupes d'émotions exprimées par chaque personne en fonction de leur valence (positive, négative, neutre ou mixte, c'est-à-dire à la fois négative et positive).

Tableau 5 : groupes d'émotions exprimées par personne selon leur valence

\begin{tabular}{|lrr|}
\multicolumn{1}{c}{} & total & $\%$ \\
\hline positives & 127 & $53 \%$ \\
neutres & 11 & $5 \%$ \\
négatives & 38 & $16 \%$ \\
mixtes & 65 & $27 \%$ \\
\hline
\end{tabular}

L'analyse plus précise des stratégies de régulation des apprentissages mobilisées par les étudiants permet de compléter notre lecture des émotions ressenties.

\subsection{Résultat 2 - Des stratégies de régulation de nature volitionnelle dominantes}

Pour recenser les stratégies de régulation des apprentissages mobilisées par les étudiants, nous avons utilisé les réponses à la question 1. L'analyse qualitative des 178 verbatim qui ont pu être codés en appliquant la taxonomie de Cosnefroy (2011) met en évidence l'importance des stratégies volitionnelles et notamment de régulation émotionnelle. Le tableau 6 recense l'ensemble des stratégies repérées dans nos verbatim. 
Tableau 6 : stratégies de régulation des apprentissages mobilisées exprimées dans les réponses à la question «Qu'avez-vous appris personnellement au cours du séminaire Esprit d'entreprendre?»

\begin{tabular}{|c|c|c|c|c|c|}
\hline & \multirow{2}{*}{\multicolumn{2}{|c|}{ nombre de verbatim exprimés }} & \multirow[b]{2}{*}{$\%$} & \multicolumn{2}{|c|}{$\begin{array}{c}\text { stratégie mobilisée } \\
\text { principalement dans le } \\
\text { dispositif }\end{array}$} \\
\hline & & & & Autodirigé & Dirigé \\
\hline \multirow[t]{4}{*}{ cognitives } & comprendre & 4 & $2 \%$ & & $\mathrm{x}$ \\
\hline & concrétiser & 3 & $2 \%$ & $\mathrm{x}$ & \\
\hline & créer des liens - créativité & 12 & $7 \%$ & $\mathrm{x}$ & \\
\hline & simplifier & 1 & $1 \%$ & $\mathrm{x}$ & \\
\hline \multirow[t]{3}{*}{ métacognitives } & $\begin{array}{l}\text { s'orienter au départ (savoirs antérieurs, contraintes de la tâche, } \\
\text { erreurs de compréhension) }\end{array}$ & 6 & $3 \%$ & & $\mathrm{x}$ \\
\hline & gérer le déroulement des tâches de traitement de l'information & 9 & $5 \%$ & & $x$ \\
\hline & élaborer les enseignements - modéliser les résultats & 10 & $6 \%$ & & $\mathrm{x}$ \\
\hline \multirow[t]{29}{*}{ volitionnelles } & contrôle volitionnel de la cognition & & & & \\
\hline & être curieux de l'avis des autres différents de soi & 19 & $11 \%$ & $\mathrm{x}$ & \\
\hline & activation d'un but d'approche & & & & \\
\hline & renforcer les buts de performance & 4 & $2 \%$ & $x$ & \\
\hline & renforcer l'instrumentalité perçue de la tâche & 12 & $7 \%$ & $\mathrm{x}$ & \\
\hline & renforcer l'importance personnelle de la tâche & 4 & $2 \%$ & $x$ & $x$ \\
\hline & oser - essayer ( proactivité) & 27 & $15 \%$ & $x$ & \\
\hline & créer un but commun à l'équipe & 8 & $4 \%$ & $x$ & \\
\hline & soutien du sentiment d'efficacité & & & & \\
\hline & activer souvenirs de réussite & 1 & $1 \%$ & $x$ & \\
\hline & fractionner en sous-tâches & 1 & $1 \%$ & $x$ & \\
\hline & auto-encouragement (on va y arriver, rien n'est impossible) & 15 & $8 \%$ & $\mathrm{x}$ & \\
\hline & contrôler les ressources disponibles & & & & \\
\hline & recherche internet, bibliothèque & 2 & $1 \%$ & $x$ & \\
\hline & diviser les tâches - déléguer pour que tous contribuent & 8 & $4 \%$ & $\mathrm{x}$ & $x$ \\
\hline & structuration du temps & & & & \\
\hline & allouer du temps et définir les priorités & 3 & $2 \%$ & $x$ & $x$ \\
\hline & définir des doses de travail optimales & 2 & $1 \%$ & $\mathrm{x}$ & \\
\hline & régulation des émotions & & & & \\
\hline & chercher le soutien / réassurance auprès d'autrui & 1 & $1 \%$ & $\mathrm{x}$ & \\
\hline & mettre de l'énergie pour convaincre les autres & 33 & $19 \%$ & $x$ & \\
\hline & vouloir être un groupe avec une forte cohésion & 9 & $5 \%$ & $\mathrm{x}$ & \\
\hline & $\begin{array}{l}\text { éviter la confrontation et la désapprobation - faire des } \\
\text { concessions }\end{array}$ & 5 & $3 \%$ & $x$ & \\
\hline & faire une présentation valorisante - surmonter sa timidité & 2 & $1 \%$ & $x$ & \\
\hline & accepter l'échec en vue de progresser & 5 & $3 \%$ & $x$ & $x$ \\
\hline & faire preuve d'empathie dans le groupe & 3 & $2 \%$ & $x$ & $\mathrm{x}$ \\
\hline & avoir confiance dans les autres & 5 & $3 \%$ & $x$ & $x$ \\
\hline & rire et/ou faire rire & 16 & $9 \%$ & $x$ & \\
\hline & $\begin{array}{l}\text { induction d'une émotion défensive (pessimisme défensif, } \\
\text { régulation introjectée) }\end{array}$ & 2 & $1 \%$ & & $\mathrm{x}$ \\
\hline défensives & $\begin{array}{l}\text { nier l'interêt du but prescrit (ça ne s'apprend pas ou ce n'est pas } \\
\text { nouveau) }\end{array}$ & 2 & $1 \%$ & & $\mathrm{x}$ \\
\hline
\end{tabular}

Nous constatons tout d'abord que les stratégies volitionnelles apparaissent plus nombreuses que les stratégies cognitives, métacognitives ou défensives. De plus, les stratégies les plus mobilisées sont toutes à but social : être curieux de l'avis des autres, faire preuve de proactivité, auto-encourager le groupe, mettre de l'énergie pour convaincre les autres, rire ou faire rire. 
Enfin, les stratégies de régulation émotionnelle y tiennent une part très importante. Toutefois le tableau met en évidence de grandes différences entre les deux dispositifs.

\subsection{Résultat 3 - Des stratégies de régulation d'apprentissage différentes selon les buts du dispositif (dirigé / autodirigé).}

Dans le dispositif dirigé, les stratégies mobilisées comportent des dimensions à la fois cognitives, métacognitives et volitionnelles. Les types de stratégies mobilisées dans le domaine cognitif (comprendre) et métacognitif (gérer le déroulement du traitement de l'information, modéliser) sont liés à la nature du projet qui fait appel à des compétences de chercheur non maîtrisées au départ par les étudiants : «J'ai appris la méthodologie de recherche, construire des hypothèses, analyser puis discuter des résultats. » "J'ai appris à mener des interviews, à les transcrire, à les coder, ce qui permet de comprendre les caractéristiques des entrepreneurs. » Pour mettre en œuvre ce projet en groupe, les stratégies liées à la coordination du travail dans le groupe restent essentielles. Lorsque les deux buts (de maîtrise des concepts et des méthodes de recherche, et d'organisation du groupe) sont atteints, alors la satisfaction est au rendez-vous. "Dans notre groupe, on était six, et nous étions très organisés. Chacun de nous avait sa fonction, comme la gestion du temps, l'apport de nouvelles idées, l'intégration de l'ensemble du travail. C'était très plaisant de travailler avec ce groupe. »

Dans le dispositif autodirigé, les stratégies apparaissent de nature différente. Au niveau cognitif, les étudiants ont cherché à concrétiser leurs idées : "Notre but était de faire passer notre concept dans la réalité, à transmettre nos idées à travers une cartographie des étapes de la création d'entreprise. » Ils ont aussi mobilisé des stratégies de créativité permettant de faire des liens entre les idées des uns et des autres au sein du groupe : «J'ai appris à ouvrir mon horizon, à investiguer de nouvelles idées, à être plus créatif, à être plus dynamique dans le cours et avec les autres. » Nous n'avons pas relevé de stratégies de nature métacognitive mais de nombreuses stratégies de nature volitionnelle. Dans un contexte de liberté créant de l'incertitude, elles visent à renforcer l'engagement au départ et à soutenir cet engagement tout au long du projet. Les étudiants ont commencé par apprendre à s'ouvrir aux autres pour trouver des idées «J'ai appris à travailler avec des personnes très différentes, qui venaient d'autres milieux et d'autres cultures. On a dû négocier ensemble ». Un grand nombre d'étudiants relève l'importance d'être proactif, c'est-à-dire d'oser, essayer, agir pour transformer les choses. «Si tu as un rêve, fais du mieux que tu peux pour le réaliser! » «a persistance c'est l'arme secrète de l'entrepreneur. Les opportunités nous font toujours signe. » «Entreprendre, ça se résume à un mot : oser. »

Pour faire preuve de cette audace, il faut mobiliser des stratégies d'auto-encouragement au sein du groupe «rien n'est impossible, ensemble on est plus fort ». Cette perception est renforcée par le désir de coopérer : "Ce qui est le plus important pour moi, c'est le sens de l'équipe. Quand on est en équipe, il y a tellement de bonnes idées qui viennent et pas de sentiments négatifs. Quand le travail est fini, je suis fier de moi et de notre groupe ». Dans ce contexte, il existe une véritable stratégie ludique (rire et faire rire) qui facilite la compréhension au sein du groupe et dans la classe : "Nous voulions mélanger l'humour et le sérieux, ce qui a permis d'atteindre le résultat que nous souhaitions », «Je pense que nous avons atteint nos objectifs 
puisque tout le monde était impliqué. Nous avons trouvé un équilibre entre l'amusement et l'apprentissage et la classe a rigolé. » Faire partager ses résultats par la classe semble avoir été un facteur de motivation extrêmement puissant pour de nombreux groupes. "Notre objectif final était de faire changer l'attitude de la classe à propos des femmes au travail. On a réussi à le faire. » «On a pu motiver la classe à travers une bonne ambiance, des bonbons et un esprit d'équipe.»

La différence entre les deux dispositifs dirigé / autodirigé est confirmée par le diagramme en radar présenté dans la figure 1. Les émotions positives sont dominantes pour les étudiants qui ont l'impression d'avoir une équipe efficace dans les deux pédagogies (traits pleins dans les deux graphiques). En revanche, les émotions sont nettement plus ambivalentes dans les groupes qui se perçoivent comme moins efficaces (traits en petits pointillés), surtout dans la pédagogie dirigée. De plus, dans la pédagogie dirigée, les émotions positives sont sensiblement moins fortes et les émotions négatives (colère, irritation et épuisement) plus présentes que dans la pédagogie autodirigée.

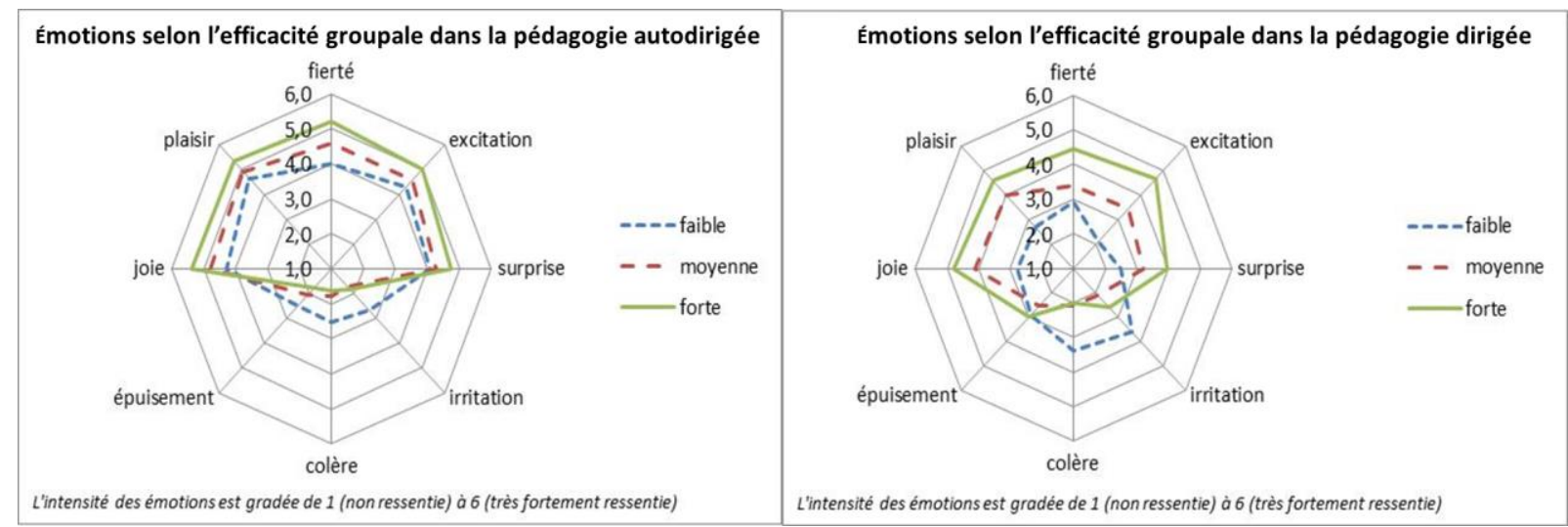

Figure 1 : Émotions selon l'efficacité groupale dans les deux dispositifs

\section{Discussion et conclusion}

Dans ce dispositif de formation à l'entrepreneuriat en groupe, notre étude exploratoire offre plusieurs contributions. La première contribution souligne le rôle des émotions positives et négatives dans la perception de l'efficacité groupale. Nous avons mesuré des corrélations modérées entre cette perception et deux groupes d'émotions : quatre positives (joie, plaisir, fierté, excitation) et trois négatives (colère, irritation et épuisement). L'étude qualitative soutient ces résultats en complétant la palette des émotions effectivement vécues et en précisant l'impact des variations émotionnelles (positives et négatives) tout au long du projet. Ainsi nous vérifions l'intuition de Cosnefroy (2011) sur la succession des émotions à valence opposée dans un épisode d'apprentissage. Les émotions constituent un indicateur fort de la capacité des groupes à s'engager et à persister dans l'effort d'apprendre.

Deuxièmement, nous confirmons les travaux de Heutté (2017) sur le versant positif : la joie et le plaisir éprouvés en groupe sont bien de l'ordre d'une "jubilation de comprendre, faire comprendre et être compris ». Mais avant d'arriver à ce niveau-là, notre analyse ajoute qu'il existe souvent une phase d'anxiété et de stress qui peut être liée à différents facteurs (incertitude 
dans une tâche très libre, incompréhension des buts du professeur, sentiment de compétence insuffisante). La qualité émotionnelle vécue au sein du groupe d'apprenants semble avoir un rôle fondamental pour aider à surmonter ces difficultés.

Ce constat nous a amenées à une troisième contribution. Nous avons en effet complété la taxonomie de Cosnefroy (2011) en identifiant différentes stratégies de régulation des apprentissages spécifiques aux groupes. Dans notre contexte de formation à l'entrepreneuriat, les stratégies les plus mobilisées par les étudiants sont de nature volitionnelle. Par ordre décroissant d'importance, ce sont les stratégies suivantes : mettre de l'énergie pour convaincre les autres, oser, être curieux de l'avis des autres différents de soi, rire et faire rire, se convaincre en groupe que rien n'est impossible, vouloir être un groupe avec une forte cohésion, créer un but commun à l'équipe, diviser les tâches pour que tous puissent contribuer, avoir confiance dans les autres, accepter l'échec en vue de progresser, faire preuve d'empathie dans le groupe.

Enfin nous avons constaté que ces différentes stratégies étaient diversement mobilisées selon les dispositifs mis en place par les enseignants. Lorsque l'enseignant adopte une posture directive, la vision des buts perçus par l'étudiant reste d'ordre académique, ce qui tend à favoriser des stratégies de régulation de type cognitif (comprendre), métacognitif et d'organisation des tâches au sein de l'équipe avec un effort d'empathie vis-à-vis des autres. Lorsque l'enseignant donne un espace de liberté supérieur, il autorise des stratégies volitionnelles plus variées au niveau des groupes. Ce qui permet de dédramatiser les difficultés et de soutenir la dynamique d'apprentissage dans le groupe et dans la classe.

Toutefois notre travail comporte plusieurs limites. Tout d'abord notre étude souffre d'un biais de mémorisation positif, lié au fait que nous avons utilisé des déclarations a posteriori. De ce fait, nous sous-estimons très probablement les stratégies défensives, beaucoup mieux étudiées par l'étude de Foliard et Le Pontois (2017) utilisant une méthodologie d'auto-confrontation croisée. D'autre part, notre étude ici ignore les dimensions de leadership dont une abondante littérature en pyscho-sociologie et comportement organisationnel montre la prégnance dans l'efficacité groupale (Hackman, 1987). Par ailleurs, notre contexte de formation à l'entrepreneuriat n'est pas neutre en termes de philosophie éducative. Elle prédispose les étudiants à préférer la démarche autodirigée qui est beaucoup plus cohérente avec l'esprit d'entreprendre. Enfin, nous n'avons pas étudié ici l'interaction entre les émotions vécues par les enseignants et celles vécues par les étudiants.

Cette étude exploratoire ouvre de nombreuses pistes de recherche ultérieure. Il serait utile de confirmer ces résultats dans d'autres contextes de travail en groupe d'étudiants, et de réaliser une observation qualitative plus fine de la variété des émotions vécues par les étudiants au fil de l'ensemble du travail de groupe. On peut supposer aussi que les groupes savent plus ou moins reconnaître et gérer les émotions selon les compétences interpersonnelles ou selon les fonctions de leadership prises en charge par les membres du groupe (Verzat et al., 2015). Si les étudiants ne sont pas en mesure de le faire dès le départ, quelles postures des enseignants ou des tuteurs pourraient aider les groupes à gérer toutes ces émotions qui les traversent ? La manière dont les différentes stratégies mobilisables peuvent être apprises est particulièrement intéressante à étudier. 


\section{Références bibliographiques}

Arpiainen, R., Lackéus, M., Taks, M. et Tynjala, P. (2013). The sources and dynamics of emotions in entrepreneurship education learning process. Trames, 17, 331-346.

Bandura, A. (1997). Self-efficacy: The Exercise of Self Control. New York: Freeman.

Barth, B.M. (1993). Le savoir en construction. Paris : Retz

Boekaerts, M. (1994). Anger in relation to school learning. Learning and instruction, 3, 269280.

Carré, P., Moisan, A. et Poisson, D. (2010). L'autoformation. Perspectives de recherche. Paris : PUF.

Cosnefroy, L. (2011). L'apprentissage autorégulé, entre cognition et motivation. Grenoble : Presses universitaires de Grenoble.

Côté, L. (2013). Améliorer ses stratégies de coping pour affronter le stress au travail. Psychologie Québec, 30(5), 41-44.

Csikszentmihalyi, M.. (1990). Flow. New York: Harper and Row.

Csikszentmihalyi, M. (2014). Flow and the Foundations of Positive Psychology. Dordrecht: Springer.

Damasio, A.R. (1995). L'erreur de Descartes. Paris : Odile Jacob.

Derouesné C. (2011). Qu'est-ce qu'une émotion ? Une introduction à l'étude des émotions. Ger Psychol Neuropsychiatr Vieil, 9(1), 69-81.

Ekman, P. (2003). Emotions revealed. New York: Times Books.

Foliard, S. et Le Pontois, S. (2017). Équipes entrepreneuriales étudiantes : comprendre pour agir. Entreprendre et Innover, 33(2), 44-54.

Guzzo, R.A., Yost, P.R., Campbell, R.J., et Shea, G.P. (1993). La puissance dans les groupes:, Articuler une construction. British Journal of Social Psychology, 32, 87-106.

Heutté, J. (2017). L'environnement optimal d'apprentissage : contribution de la recherche empirique sur les déterminants psychologiques de l'expérience positive subjective aux sciences de l'éducation et de la formation des adultes. Sciences et bonheur, 2, 82-99.

Hinton, C., Miyamoto, K. et Della-Chiesa, B. (2008). Brain research, learning and emotions: Implications for education research, policy and practice. European Journal of Education, 43(1), 87-103.

Ionescu, S., Jaquet, M.M. et Lhote, C. (1997). Les mécanismes de défense. Théorie et Clinique. Paris : Nathan University.

Johnson, D.W. et Johnson, R. (1991). Learning together and alone: Cooperation, competition and individualization (3rd edition). Englewood Cliffs, NJ: Prentice Hall.

Johnson, D.W. et Johnson, R. (1998). Un survol de l'apprentissage coopératif. Dans Thousand, L., Villa, R.A. et A.I. Nevin, La créativité et l'apprentissage coopératif(p. 103-133). Montréal : Les Éditions Logiques.

Knowles, M.S. (1975). Self-Directed Learning : A guide for learners and teachers. Cambridge: Prentice Hall.

Locke, K. (2001). Grounded theory in management research. London : Sage Publications. 
Matlin, M.W. et Stang, D.J. (1978). The Pollyanna Principle: Selectivity in Language, Memory, and Thought. Cambridge : Schenkman Pub. Co.

Morris, M.H., Jeffrey A.A., Kuratko, D.F. et Brannon, D. (2010). Experiencing family business creation: Differences between founders, nonfamily managers, and founders of nonfamily firms. Entrepreneurship Theory and Practice, 34(6), 1057-1084.

Pekrun, R. (2006). The value-control theory of achievement emotions: assumptions, corollaries, and implications for educational research and practice. Educational Psychology Review, 1(4), 310-341.

Pekrun, R., Frenzel, A.C., Goetz, T. et Perry, R.P. (2002). Academic Emotions in Students' Self-Regulated Learning and Achievement: A Program of Qualitative and Quantitative Research. Educational Psychologist, 37(2), 91-105.

Pekrun, R. (2014). Emotions and Learning. Educational Practices Series. Belley: Gonnet.

Pekrun, R., Frenzel, A. C., Goetz, T. et Perry, R.P. (2007). The control-value theory of achievement emotions: An integrative approach to emotions in education. Dans P. Schutz et R. Pekrun (Eds), Emotion in education (p. 13-36). Amsterdam: Elsevier Academic Press.

Peyrat-Malaterre, M.F. (2011). Comment faire travailler efficacement des élèves en groupe? Tutorat et apprentissage coopératif. Paris : De Boeck.

Pittaway, L. et Cope, J. (2007). Entrepreneurship education. International Small Business Journal, 25(5), 479-510.

Rogers, C.R. (1972). Liberté pour apprendre (traduction française Freedom to learn). Paris : Dunod.

Roschelle, J. et Teasley, S.D. (1995). The construction of shared knowledge in collaborative problem solving. Dans C. O'Malley (Ed.), Computer Supported Collaborative Learning (p. 6997). Berlin: Springer-Verlag.

Rosenberg, M. (1999). Les mots sont des fenêtres ou bien ce sont des murs, introduction à la communication non violente. Paris : La Découverte et Syros.

Schutz, P. et Pekrun, R. (Eds). (2007). Emotion in Education. Amsterdam: Elsevier Academic Press.

Valiente, C., Swanson, J. et Eisenberg, N. (2012). Linking students' emotions and academic achievement: When and why emotions matter? Child Development Perspective, 6(2), 129-135.

Verzat, C., O’Shea, N. et Jore, M. (2016). Rendre les étudiants heureux et proactifs grâce à l'apprentissage autodirigé. Entreprendre et Innover, 29(2), 37-48.

Verzat, C., O'Shea, N. et Jore, M. (2017). Teaching proactivity in the entrepreneurial classroom. Entrepreneurship \& Regional Development, 29(9-10), 975-1013.

Zimmerman, B.J. (1990). Self-regulated Learning and Academic Achievement: An Overview. Educational Psychologist, 25(1), 3-17. 